\title{
Funktionelle Dyspepsie oder Gastroparese?
}

Die Symptome der funktionellen Dyspepsie und einer Gastroparese überlappen sich. Für den Therapieerfolg ist daher die Ermittlung der zugrundeliegenden Pathophysiologie nötig.

Sodbrennen, postprandiales Völlegefühl, Übelkeit und Erbrechen - solche und weitere Symptome können sowohl auf eine funktionelle Dyspepsie als auch auf eine Gastroparese hinweisen. Die Gastroparese zeichnet sich durch eine verzögerte Magenentleerung aus, ohne dass eine mechanische Obstruktion vorliegt. Bei einer funktionellen Dyspepsie könne zusätzlich die Magenentleerung beschleunigt sein oder eine hypersensitive gastrische oder duodenale Reaktion auf Dehnungsreize oder Nahrungsmittel vorliegen, erklärte Prof. Dr. Michael Camilleri, Rochester,
Minnesota. Für eine erfolgversprechende Behandlung empfahl der Gastroenterologe daher zunächst die schrittweise Differenzialdiagnostik.

\section{Obstruktion ausschließen}

Zunächst müsse eine mechanische Obstruktion ausgeschlossen werden, üblicherweise endoskopisch oder per CT- oder MRT-Enterographie. Zweitens sollten iatrogene Ursachen einer Gastroparese in Betracht gezogen werden. Dazu gehört die Einnahme von Opiaten. Ebenso können verschiedene Antidiabetika wie GLP1-Agonisten oder Gliptine die Magenentleerung verzögern. „5-12\% der Patienten mit Diabetes berichten über Symptome, die auf eine Gastroparese zurückgeführt werden können“, sagte Camilleri. Entzugserscheinungen bei Cannabisgebrauch gehen unter Umständen mit Hyperemesis einher. Schließlich sind auch Verletzungen des Nervus vagus bei Operationen möglich. Gegebenenfalls ist eine Magenentleerungs-Szintigrafie angezeigt.

Die häufigsten Ursachen einer Gastroparese sind nach Camilleris Angaben neuropathische Störungen wie bei Diabetes mellitus, nach Vagotomie oder bei Myopathien. Behandelt wird dann in erster Linie mit Prokinetika und Antiemetika. Bei Dyspepsie mit normaler Magenentleerung und reduzierter Nahrungstoleranz werden außer diätetischen Änderungen, Protonenpumpeninhibitoren und Prokinetika neuerdings auch trizyklische Antidepressiva oder 5HT1A-Agonisten wie Buspiron empfohlen.

Falk Symposium 200 "Therapeutic Strategies in Diseases of the Digestive Tract 2015 and Beyond", Oktober 2015, Freiburg

\section{Nekrotisierende Pankreatitis: Konservative Therapie bevorzugt}

\section{Die Zahl offener Operationen we- gen nekrotisierender Pankreatitis ist in den vergangenen Jahren dra- matisch gesunken. Wann immer möglich, wird heute konservativ behandelt.}

Verfolgt werde eine schrittweise Eskalation der Therapieinvasivität, erklärte Prof. Dr. Thilo Hackert, Heidelberg. So lange wie möglich wird versucht, den Patienten mit akuter nekrotisierender Pankreatitis mit intensivtherapeutischen Maßnahmen zu helfen. Ein Kernkriterium der Ent- scheidungsfindung sei die Frage, ob eine infektiöse Pankreasnekrose vorliege. In diesem Fall müsse das Fortschreiten der Erkrankung und eine Sepsis verhindert werden. Empfohlen wird dazu üblicherweise, eine Feinnadelpunktion des Pankreas vorzunehmen, um mikrobiologische Untersuchungen vornehmen zu können. In der Praxis spiele dies jedoch eine untergeordnete Rolle, so Hackert, da bei einem entsprechendem klinischen Bild sowieso eine perkutane Spüldrainage gelegt werde, über die dann Material gewonnen werden kann.

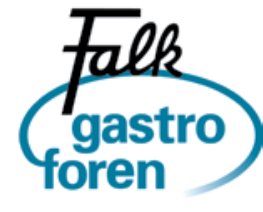

Besuchen Sie das nächste Falk Gastro Forum

"Choosing wisely - Standards und Kontroversen in der viszeralen Medizin" am Samstag, den 23. Januar 2016 in Mannheim; Infos: www.drfalkpharma.de/veranstaltungen
Lässt sich der infektiöse Herd nicht beseitigen, kommen die endoskopische $\mathrm{Ne}$ krosektomie oder die minimal-invasive transabdominelle Nekrosektomie in Betracht. Die endoskopische Nekrosektomie ist allerdings nicht überall verfügbar. Postoperativ erfolgt über Drainagen eine kontinuierliche Lavage.

Die Erfolgsraten beider Vorgehensweisen seien vergleichbar und die Prognose deutlich besser als nach offener Laparotomie, so Hackert. Die offene Operation gilt wegen der deutlich höheren Mortalitätsrate inzwischen als ultima ratio und wird nur vorgenommen, wenn intraabdominale Komplikationen auftreten wie Darmperforationen oder schwere Blutungen.

(TM)

Falk Symposium 200 "Therapeutic Strategies in Diseases of the Digestive Tract 2015 and Beyond", Oktober 2015, Freiburg 